\title{
Critical insight into the influence of the potential energy surface on fission dynamics
}

\author{
K. Mazurek, ${ }^{1,2, *}$ C. Schmitt,${ }^{2}$ J. P. Wieleczko, ${ }^{2}$ P. N. Nadtochy ${ }^{3}$ and G. Ademard ${ }^{2}$ \\ ${ }^{1}$ The Niewodniczański Institute of Nuclear Physics - PAN, 31-342 Kraków, Poland \\ ${ }^{2}$ Grand Accélérateur National d'Ions Lourds (GANIL), CEA/DSM-CNRS/IN2P3, 14076 Caen, France \\ ${ }^{3}$ Omsk State University, Department of Theoretical Physics, 644077 Omsk, Russia
}

(Dated: May 17, 2011)

\begin{abstract}
The present work is dedicated to a careful investigation of the influence of the potential energy surface on the fission process. The time evolution of nuclei at high excitation energy and angular momentum is studied by means of three-dimensional Langevin calculations performed for two different parametrizations of the macroscopic potential: the Finite Range Liquid Drop Model (FRLDM) and the Lublin-Strasbourg Drop (LSD) prescription. Depending on the mass of the system, the topology of the potential throughout the deformation space of interest in fission is observed to noticeably differ within these two approaches, due to the treatment of curvature effects. When utilized in the dynamical calculation as the driving potential, the FRLDM and LSD models yield similar results in the heavy-mass region, whereas the predictions can be strongly dependent on the PES for medium-mass nuclei. In particular, the mass, charge and total kinetic energy distributions of the fission fragments are found to be narrower with the LSD prescription. The influence of critical model parameters on our findings is carefully investigated. The present study sheds light on the experimental conditions and signatures well suited for constraining the parametrization of the macroscopic potential. Its implication regarding the interpretation of available experimental data is briefly discussed.
\end{abstract}

PACS numbers: 25.70.Jj, 24.75.+i, 25.85.-w, 47.55.D-, 21.10.Ft

\section{INTRODUCTION}

The description of the potential energy plays a crucial role in the predictions of the structural properties of the nucleus as well as in the understanding of the dynamics of nuclear reactions. The Potential Energy Surface (PES) defines the evolution of the energy of a nucleus as a function of its shape deformation. As such, PES calculations are important to determine various nuclear properties, including ground-state masses, excited particlehole configurations, collective features from low to high spin, shape isomerism, fission barriers, etc. The potential energy can be calculated in a microscopic scheme using self-consistent models based on the Hartree-Fock approach [1-3]. Yet, the realistic description of the variety of possible nuclear shapes requires considering a multi-dimensional deformation space, and fully microscopic calculations become computing-wise prohibitive with increasing the number of dimensions. An alternative approach for a reliable calculation of the potential energy landscape is based on the phenomenological macroscopic-microscopic method (see Ref. [4] and therein). In there, the potential energy is determined as the sum of shape-dependent macroscopic and microscopic (shell-plus-pairing) terms. The foremost advantage of this approach relies on its simplicity and high flexibility, allowing fast predictions in a highly multi-dimensional deformation space over the whole nuclear chart $[5,6]$. The transparent physical meaning of the parameters adds

*Electronic address: mazurek@ifj.edu.pl to its attractiveness. The macroscopic-microscopic approach appeared to be very powerful in describing various phenomena related to structural and dynamical aspects. As for some recent salient and appealing results, we mention shape coexistence in medium-mass nuclei [5], hyper-deformation [7], half-lives of super-heavy nuclei [8] and giant-dipole resonance strength functions [9].

There exists several models for calculating both the macroscopic and the microscopic contributions to the potential energy. The former is customarily computed within a Liquid-Drop-like formalism, while the latter is usually derived following the method proposed by Strutinsky [10]. The microscopic contribution is crucial for describing ground-state and low-energy properties, and it vanishes at high temperature and/or angular momentum. The potential surface is then solely determined by the macroscopic component (including the rotational energy). The present work is dedicated to a detailed survey of the macroscopic part of the PES and its different terms.

The pioneering work on the macroscopic potential energy is to be attributed to Bethe and Weizsäcker $[11,12]$ who, soon after the discovery of fission in 1935, proposed to envisage the atomic nucleus as a spherical charged liquid drop. With such a parallel, the potential energy is built of a volume, a surface and a Coulomb term. Several refinements of this early derivation have emerged over the years. The major improvements have been made by Myers and Swiatecki [13-15] who developed the well-known and powerful deformationdependent Liquid Drop Model (LDM), and further by Cohen, Plasil and Swiatecki [16] with the inclusion of rotation of the system in the so-called Rotating Liquid 
Drop Model (RLDM). The RLDM formula was revisited and an additional term accounting for curvature effects was introduced [17-19]. This latest version of the LDM formula is referred to as the Lublin-Strasbourg Drop (LSD) parametrization. The coefficients of the LSD prescription were adjusted to reproduce most recent data on nuclear masses [20]: An unprecedented accuracy of $0.623 \mathrm{MeV}$ (RMS) was achieved. The LSD formula was also found to yield an improved description of available experimental fission barriers [21]. In parallel to these developments, Sierk and collaborators [22, 23] succeeded in deriving a formulation which accounts for the finite range of nuclear forces, nuclear saturation and diffusivity of the nuclear surface. The resulting Finite Range Liquid Drop Model (FRLDM) is somehow more complex than the aforementioned prescriptions because the calculation of the surface term requires the double folding of Yukawa functions over the nuclear volume. Yet, it is still convenient to use, and FRLDM is probably the Liquid-Drop-based formulation most used in contemporary calculations. The latest set of its parameters [24] describes experimental masses with a precision of $0.752 \mathrm{MeV}$ (RMS) and fits reasonably well fission barriers. Besides its well-established performances and specific assets, the parametrization by Sierk et al. [22, 23] does not explicitly include curvature effects as the LSD model does. To our knowledge, a meticulous investigation of the importance of curvature effects on nuclear dynamics is still lacking. Such a study is proposed to be undertaken here.

As a typical large-scale amplitude collective motion, fission is an ideal research laboratory for probing the potential energy landscape. In fission a nucleus evolves from a ground-state shape to the configuration of two separated fragments. Nuclei undergoing fission can thus be described by a multi-dimensional potential energy surface that guides the shape evolution. Yet, although the time evolution of the hot rotating nucleus is sensitive to the details of the PES, the dynamical evolution depends as well on the inertia of the system and energy damping mechanisms. To enhance the sensitivity to the PES requires a suitable choice of the system. Fission of medium-mass nuclei is very relevant in this respect. Indeed, while heavy nuclei mainly exhibit ellipsoidal shapes along their path to fission, lighter systems have to be particularly deformed and/or necked-in to reach scission. The saddle point shape already likely resembles two deformed spheroids separated by a well-developed neck. The accurate parametrization of all the details of the PES is therefore expected more critical for describing the fission of medium-mass nuclei as compared to heavy systems. In particular, an appropriate modelling of surface and curvature effects is conjectured to be important. Besides the reaction mechanism and the mass of the system, the choice of the experimental observable is critical as well.
The present work is dedicated to a study of the influence of the potential energy landscape on the decay of hot rotating medium-mass nuclei. State-of-the-art three-dimensional dynamical Langevin calculations are performed using two different prescriptions of the PES, namely the FRLDM and the LSD model. The relevance of different observables in their ability to constrain the parametrization of the macroscopic potential entering into the modelling of fission is investigated. Fission-fragment mass, charge and total kinetic energy distributions will reveal to be particularly well suited signatures. While the influence of various ingredients of the Langevin equations, like e.g. the level density and the friction strength, has been widely studied (see Ref. [25-30] and therein), investigations on the sole influence of the PES parametrization are scarce. For fission of nuclei with $A \geq 200$, Gontchar et al. [31] analysed the results of two-dimensional Langevin calculations while varying several ingredients of the model, among which the parametrization of the potential energy. They noticed that, depending on the excitation energy and the observables studied, RLDM [13] and FRLDM [4, 22] can yield very different results. Recently, a careful study on the influence of the shape of the potential on the fission rate was presented in Ref. [32]. The calculation restricted to one dimension and very schematic potentials are tested, only.

The moderate fissility of medium-mass systems makes theoretical and experimental investigations challenging. From the theoretical point of view, previous investigations showed that the results, regarding various observables, depend on the dimension of the calculation [27, 3437]. As such, realistic calculations, able to predict the correlation between observables, require to be performed in a multi-dimensional deformation space, what demands high computing time. Experimentally, high efficiency and precision are mandatory due to the low fission crosssection involved. To our knowledge, one work [38] of this kind exists, only. The main focus was on friction and no test of the PES was performed. The present work is dedicated to pin down the sole influence of the energy landscape. Some preliminary results have been reported in [39]. The paper is organized as follows: In Section II the model is presented with emphasis on the ingredients of importance for the present concern. The results are gathered in Section III where static and dynamical considerations are detailed separately. Our concluding remarks are given in Section IV.

\section{DYNAMICAL MODEL AND INGREDIENTS}

A purely microscopic description of fission is a challenge, still today $[40,41]$. It is therefore customary to describe the decay of hot rotating compound nuclei by the use of hybrid models, namely transport theories which distinguish between collective and intrinsic de- 
grees of freedom (see Ref.[42] and therein). Intrinsic excitations are based on the states of the individual constituents, while collective modes correspond to a coordinated motion involving most of the constituents. Within Kramers' seminal picture [43], the collective modes can be viewed as Brownian particles interacting stochastically with a heat bath. The evolution of the system is given by the solution of either the FokkerPlanck or the Langevin classical equation of motion, where the combined action of the driving potential, friction and diffusion forces is computed, determining the trajectory of the nucleus on the PES. While the (differential) Fokker-Planck equation gives access to distribution probabilities as a function of time, the (integral) Langevin equation permits to trace the time evolution of the system step by step for individual trajectories. The present work uses the latest version of the three-dimensional Langevin model developed by Adeev and collaborators [27, 44, 45]. This code showed successful in describing experimental data on heavy-ion induced fission over a wide range in mass and energy $[27,29,30,45-48]$. The features of the model, most relevant for the present concern, are given below. We refer the reader to the quoted literature for further details.

The dynamical evolution of the hot rotating system in the three-dimensional potential energy landscape is obtained by solving the following coupled Langevin equation

$$
\begin{aligned}
\frac{d q_{i}}{d t} & =\sum_{j} \mu_{i j}(\vec{q}) p_{j} \\
\frac{d p_{i}}{d t} & =-\frac{1}{2} \sum_{j, k} \frac{d \mu_{i j}(\vec{q})}{d q_{i}} p_{j} p_{k}-\frac{d F(\vec{q})}{d q_{i}}-\sum_{j, k} \gamma_{i j}(\vec{q}) \mu_{i j}(\vec{q}) p_{k}+\sum_{j} \theta_{i j}(\vec{q}) \Gamma_{j}(t)
\end{aligned}
$$

where $\vec{q}=\left(q_{1}, q_{2}, q_{3}\right)$ is the vector of collective coordinates and $\vec{p}$ the corresponding conjugate momentum. The collective coordinates are closely related to the choice of the parametrization of the shape of the system. In the present model, the "funny hills" $(c, h, \alpha)$ parametrization [49] was adopted as it has shown to be able to describe in a realistic way the large variety of shapes that a nucleus may take along its path to fission. For reason of convenience [47], the collective variables $\left(q_{1}, q_{2}, q_{3}\right)$ entering eq.(1) do not correspond to the very original "funny hills" coordinates, but are derived from them according to

$$
\begin{aligned}
q_{1} & =c \\
q_{2} & =\frac{h+3 / 2}{\frac{5}{2 c^{3}}+\frac{1-c}{4}+3 / 2} \\
q_{3} & = \begin{cases}\alpha /\left(A_{s}+B\right), & B \geq 0 \\
\alpha / A_{s}, & B<0\end{cases}
\end{aligned}
$$

where the $A_{s}$ and $B$ are defined as

$$
B=2 h+\frac{c-1}{2}
$$

For $B \geq 0$ dashed

$$
A_{s}=c^{-3}-\frac{B}{5}
$$

For $B<0$

$$
A_{s}=-\frac{4}{3} \frac{B}{\exp \left(B c^{3}\right)+\left(1+\frac{1}{2 B c^{3}}\right) \sqrt{-\pi B c^{3}} \operatorname{erf}\left(\sqrt{-B c^{3}}\right)}
$$

The coordinates $\left(q_{1}, q_{2}, q_{3}\right)$ are connected to the elongation, neck thickness and mass asymmetry of the nucleus, respectively. The driving potential is given by the Helmholtz free energy $F(\vec{q})=V(\vec{q})-a(\vec{q}) T^{2}$ with $V(\vec{q})$ being the bare potential energy (see below). A Fermi-gas model is assumed for the determination of the temperature according to $T=\sqrt{E_{\text {int }} / a(\vec{q})}$ where $E_{\text {int }}$ and $a(\vec{q})$ are the intrinsic excitation energy and level-density parameter, respectively. Several prescriptions can be used for the latter, among which the deformation-dependent expression proposed by Ignatyuk [50] which is assumed by default in the code. The mass tensor $m_{i j}(\vec{q})\left(\left\|\mu_{i j}\right\|=\right.$ $\left.\left\|m_{i j}\right\|^{-1}\right)$ is calculated within the Werner-Wheeler approximation for incompressible irrotational flow [51] and the friction tensor $\gamma_{i j}(\vec{q})$ is derived assuming a one-body dissipation mechanism $[52,53]$ with the possibility of reducing its strength by means of a factor denoted $k_{s}$ $[27,45]$. The last term in the right-hand side of eq.(1) is related to the diffusion tensor $D_{i j}(\vec{q})=\theta_{i k} \theta_{k j}$ derived from Einstein's relation $D_{i j}(\vec{q})=\gamma_{i j}(\vec{q}) T$, where $\theta_{i k}$ is the random force strength tensor. The stochastic nature of the diffusion process is accounted for by the normalized Gaussian white noise term $\Gamma_{j}(t)$.

The initial conditions of the system are assumed to correspond to a spherical compound nucleus with a total excitation energy $E^{\star}$ given by the entrance-channel of the reaction. The angular momentum $L$ for each Langevin trajectory is sampled from a triangular distribution function which maximum is given by the critical angular momentum $L_{\text {crit }}$ for fusion [25]. The initial conditions in momentum assume thermal equilibriumdashed [27]. 
De-excitation of the system by evaporation of light particles $(n, p, \alpha)$ is taken into account along the path to fission using a Monte-Carlo approach [25]. The decaywidth for the emission of a given particle is calculated with an updated version of the statistical code Lilita [54] based on Hauser-Feschbach's theory [55]. For the present work, particle transmission coefficients are assumed deformation-independent. Post-scission evaporation (i.e. by the fission fragments) is not evaluated here. It would noticeably increase the computing time, while not affecting the conclusions of our work. Besides, we mainly concentrate on fission-fragment charge distributions which can be assumed as not influenced by this simplification due to the very small probability of chargedparticle (as compared to neutron) emission by the fragments after scission.

During a random walk along the trajectory in the collective coordinate space, energy conservation is ensured according to $E^{\star}=E_{\text {int }}+E_{\text {coll }}+V(\vec{q})+E_{\text {evap }}(t)$, where $E_{\text {coll }}$ is the kinetic energy of the collective motion (including the rotational energy), and $V(\vec{q})$ is the potential energy at the actual point of the trajectory on the PES at time $t$. The energy carried away by particle evaporation at that time is accounted for by $E_{\text {evap }}(t)$. Note that the collective and potential energies implicitly depend on the angular momentum. Scission is defined by the criterion of a finite neck radius $R_{N}=0.3 R_{0}$ with $R_{0}$ is the radius of the corresponding compound nucleus [56].

Each trajectory is simulated dynamically by solving the Langevin equation up to a certain time. A transition to a statistical branch is implemented in the code for limiting the computing time [25]. This transition occurs under appropriate conditions i.e. provided the nucleus, which has lost a significant part of its initial excitation energy, is still near the ground state and the fission rate has reached its asymptotic limit. At that stage of the simulation, the decay is computed by the conventional statistical model approach. If, after the transition to the statistical branch, the nucleus undergoes fission, the code switches again to the dynamical treatment in order to determine the trajectory of the system between saddle and scission.

As outlined in introduction, the ingredient of the Langevin equation of main interest in the present work is the potential energy. Two sets of calculations are performed, assuming for $V(\vec{q})$ either the FRLDM [4, 22, 23] or the LSD [17] prescription. In this work a term accounting for the deformation-dependent congruence energy [57] is added to the early LSD formula [17], as it was shown to improve the description of experimental fission barriers in medium-mass nuclei [21]. Along the remainder of the present paper, the acronym LSD includes this congruence term. It should be emphasized that the FRLDM potential is adopted in most of the contemporary Langevin calculations, while the LSD potential was so far used in one work only, and within a one-dimensional model [33].
The presently used Langevin code gives access to many quantities and correlations that are available in experiment $[27,28,45,47]$. Here we concentrate on fission probabilities, pre-scission particle multiplicities and fission-fragment mass, charge and total kinetic energy distributions. These observables are foreseen as most sensitive [45] to the phenomenon of interest. The analysis of other quantities such as fission-fragment anisotropies and light-particle energy spectra, also available from the theory, is in progress.

\section{RESULTS}

As explained previously, the influence of the topology of the PES, and namely the parametrization of curvature effects, is expected strongest for medium-mass fissioning systems. This work focuses on the de-excitation of hot rotating ${ }^{118} \mathrm{Ba}$ compound nuclei, recently investigated experimentally in inverse kinematics [58] with the reaction ${ }^{78} \mathrm{Kr}+{ }^{40} \mathrm{Ca}$ at bombarding energy $E_{l a b}=429 \mathrm{MeV}$. The total excitation energy of the compound nucleus amounts to $E^{\star}=98.8 \mathrm{MeV}$ and spins up to $L_{\text {crit }}=70 \hbar$ are predicted to be populated. In order to probe the sensitivity of the dynamical evolution to the profile of the PES as a function of the size of the system, we study in parallel the fission of a very heavy nucleus. As a reference point, calculations are made for the reaction ${ }^{20} \mathrm{Ne}+{ }^{240} \mathrm{Pu}$ at $E_{l a b}=142 \mathrm{MeV}$ [59] producing ${ }^{260} \mathrm{Rf}$ compound nuclei with $E^{\star}=74.2 \mathrm{MeV}$ and $L_{\text {crit }}=50 \hbar$. This system was partly investigated within the present approach in Ref. [27].

The fate of the initially hot and rotating nucleus is determined by its dynamical evolution on the top of the static potential energy landscape. In the following we first focus on the results of the calculation for the static properties of the system. These are of help for analyzing the dynamical results presented in a second step, and understanding possible differences depending on the PES used.

\section{A. Topology of the potential energy surface}

In Fig. 1 the potential energy landscape obtained with the FRLDM (top) and the LSD (bottom) formula is displayed in the two-dimensional $\left(q_{1}, q_{2}\right)$ deformation subspace assuming $\alpha=0.0$ for the ${ }^{260} \mathrm{Rf}$ compound system at $L=0 \hbar$. Dashed lines indicate the mean path to fission. Although the tendency is the same for both parametrizations (i.e. valley towards large $q_{2}$ with $q_{1} \sim$ 1.8 and barrier in the $q_{1}$ direction for $q_{2} \sim 0.6$ ), the profile of the landscape is more soft for FRLDM.

A similar comparison of the potential energy surfaces is shown in Fig. 2 for the ${ }^{118} \mathrm{Ba}$ compound nucleus at $L=56 \hbar$. For this system, the PES topologies obtained with the FRLDM and LSD models noticeably differ. While a single valley is observed for LSD along 
$q_{2} \sim(0.6-1.0)$, two valleys develop with FRLDM: the path labelled "1" follows the same direction than the one obtained with LSD, while an additional path labelled "1a" goes towards large $q_{1}$ for $q_{2} \sim 0.6$. Valley "1a" observed with FRLDM corresponds to lemon-like shapes which can not lead to a binary fission. This artificial path disappears with the LSD prescription owing to a proper account of curvature effects (which are missing in FRLDM). Besides the occurrence of valley "1a", one also observes a sizeable difference between the two PES in terms of the stiffness of the landscape. This difference is at variance with what obtained for the ${ }^{260} \mathrm{Rf}$ system. We shall note that the stiffness of the PES depends on the angular momentum. In Fig. 2 we deliberately consider for the ${ }^{118} \mathrm{Ba}$ compound nucleus a high value of $L$ for which the contribution to fission is large. Yet, the observation of markedly different stiffnesses between FRLDM and LSD for this medium-mass nucleus, in contrast to ${ }^{260} \mathrm{Rf}$, remains valid down to $L=0 \hbar$.

The topology of the PES along the asymmetry coordinate plays a crucial role in determining the fission partition (see Ref. [60] and therein). The deformation of the actual saddle point can thus not be established on the $\left(q_{1}, q_{2}\right)$ subspace alone. The third (asymmetry) coordinate is considered in Fig. 3 where the ${ }^{118} \mathrm{Ba}$ potential energy surface is shown for both PES parametrizations in the $\left(q_{1}, q_{3}\right)$ subspace assuming $h=0.0$. The mean symmetric (valley "1") and asymmetric (valley "2") fission trajectories are indicated by solid lines. Again, for the ${ }^{118} \mathrm{Ba}$ system, the valleys are observed to be characterized by notably different stiffnesses depending on the prescription used for the PES. For the ${ }^{260} \mathrm{Rf}$ compound nucleus, the difference in the $\left(q_{1}, q_{3}\right)$ subspace (not shown) is less, as it is the case in the $\left(q_{1}, q_{2}\right)$ subspace discussed previously. All together, according to static considerations, one may expect a stronger influence of the PES prescription for the fission of medium-mass nuclei as compared to heavy systems. In particular the difference observed between the FRLDM and LSD profiles in Fig. 3 at, and beyond, the saddle point $\left(q_{1} \sim 1.8\right)$ suggests sizeable different predictions for the fission-fragment mass and charge distributions.

Due to the moderate fissility of ${ }^{118} \mathrm{Ba}$, the amount of angular momentum brought into the system by the reaction is decisive regarding the fate of the excited compound nucleus. The evolution of the symmetric and asymmetric fission barrier $B_{f}$ with angular momentum is displayed in Fig. 4 using either the FRLDM or the LSD prescription. The height of the barrier is taken as the difference between the potential energy at the relevant saddle and equilibrium points. For each $L$ the symmetric barrier was obtained after minimisation of the potential energy in the two-dimensional $\left(q_{1}, q_{2}\right)$ subspace with the constraint $\alpha=0$ (along trajectory "1" in Fig. 3). The asymmetric barrier (any $\alpha \neq 0$ ) was extracted from the most probable path in the $\left(q_{1}, q_{3}\right)$ subspace assuming $h=0$ (along trajectory "2" in Fig. 3). This is justified because $h=0$ approximately follows the bottom of the
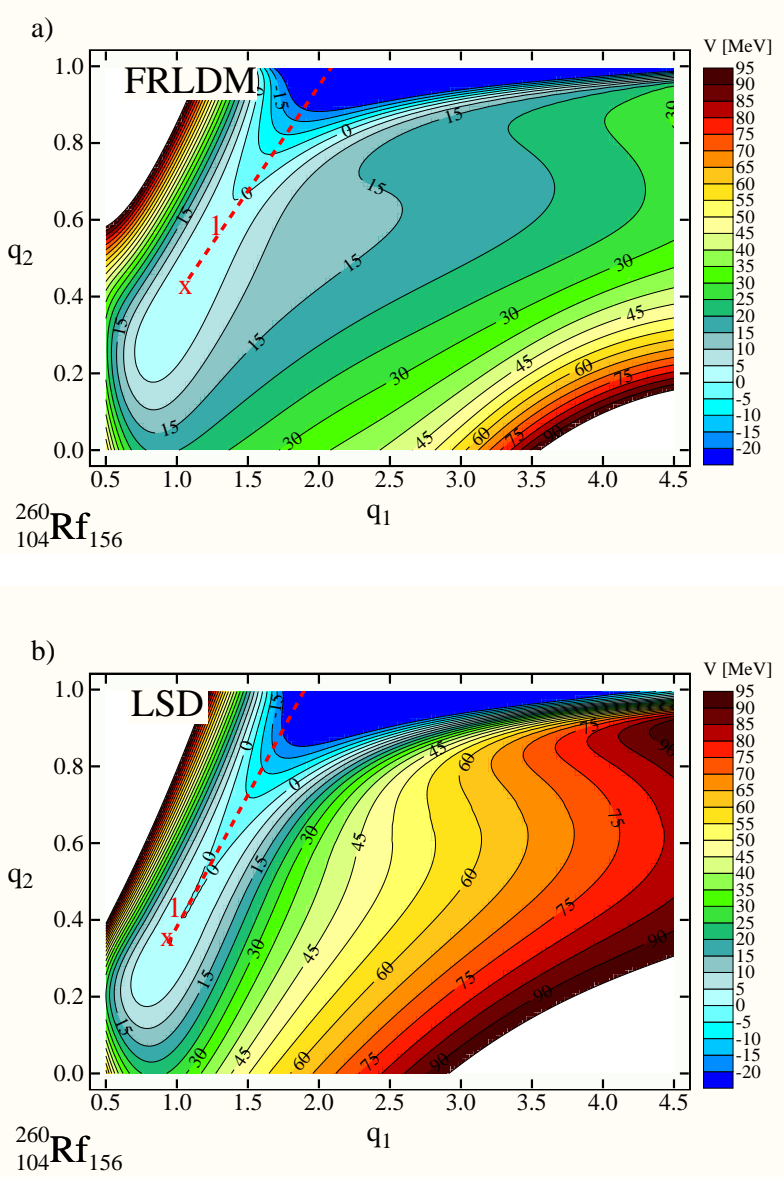

FIG. 1: (colour on-line) Potential energy surface for the ${ }^{260} \mathrm{Rf}$ compound nucleus with $L=0 \hbar$ in the plane $\left(q_{1}, q_{2}\right)$ for $\alpha=$ 0.0 as obtained with the FRLDM (a) and the LSD (b) model. The red dashed lines follow the mean path to fission.

fission valley $[31,49]$. The evolution of the barrier with $L$ is observed to be independent of the PES parametrization: At low angular momentum, the symmetric barrier exceeds the asymmetric one, and this trend gets reversed with increasing spin. The angular momenta at which symmetric and asymmetric barrier heights are equal are nevertheless different, amounting to $L \sim 58 \hbar$ and $\sim 48 \hbar$ for the FRLDM and LSD model, respectively. The calculated $B_{f}$ values are generally larger for the LSD formula over the whole $L$ range, although the FRLDM and LSD symmetric barriers become very close above $L \sim 28 \hbar$.

Based on the static considerations of Fig. 4, a transition from asymmetric to symmetric mass (equivalently, charge) partition is expected for fission of ${ }^{118} \mathrm{Ba}$ with increasing spin. A parallel may be drawn between this conjecture and the Businaro-Gallone transition [61]. The value $x_{B G}$ of the fissility parameter $x$ [16] at which the Businaro-Gallone point is located is difficult to extract precisely from any such calculation due to the flatness of 

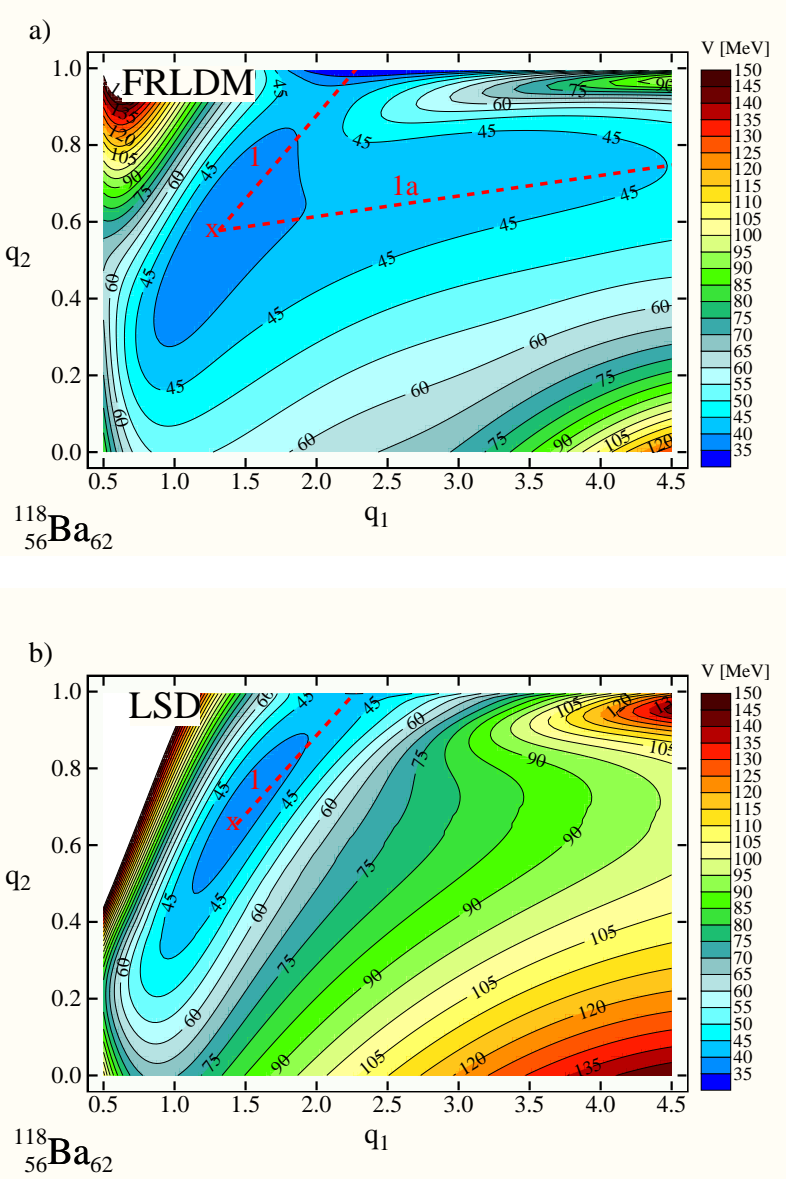

FIG. 2: (colour on-line) Identical to Fig. 1 for the ${ }^{118}$ Ba compound nucleus with $L=56 \hbar$.

the PES around that point $[62,63]$. The experimental determination of $x_{B G}$ is not easy neither. Due to the dependence of its location on $L$ and the fact that usually a wide range of $L$ is populated in a nuclear collision, no net transition from asymmetric to symmetric fission in terms of $x$ could be evidenced in experiment so far $[64,65]$. The fissility of ${ }^{118} \mathrm{Ba}$ is expected to lie close to $x_{B G}$ and, according to Fig. 4, we may expect a bifurcation of the system from the asymmetric to the symmetric fission valley with increasing $L$. That could be seen as a "Businaro-Gallone-like transition" as a function of $L$.

\section{B. Dynamical results}

The results of the previous section support the intuitive idea about the relevance of studying fission of mediummass nuclei to bring out the influence of the detailed topology of the PES. A suited choice of the system is nonetheless not sufficient, and an observable, which sen-
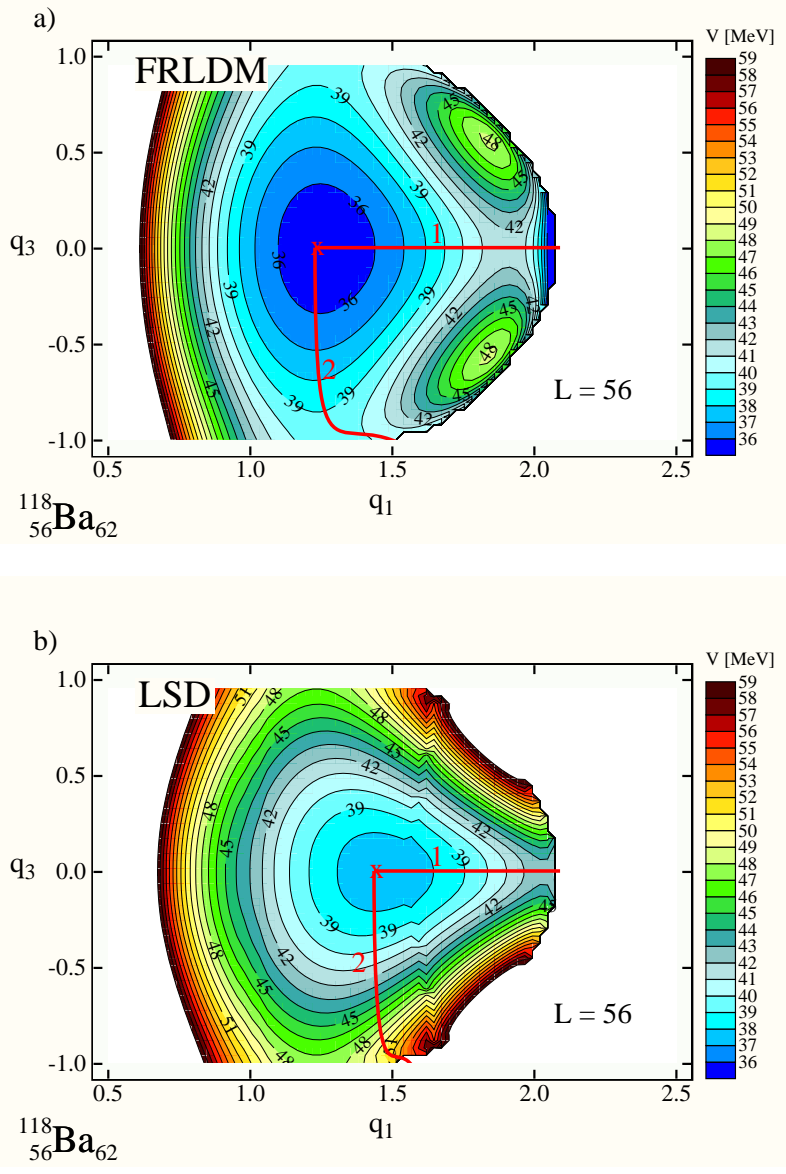

FIG. 3: (colour on-line) Potential energy surface for the ${ }^{118} \mathrm{Ba}$ compound nucleus with $L=56 \hbar$ in the plane $\left(q_{1}, q_{3}\right)$ for $h=0.0$ as obtained with the FRLDM (a) and the LSD (b) model. The red solid lines follow the mean symmetric ("1") and asymmetric ("2") path to fission.

sitivity to the PES is particularly strong, is required. In the present section, we analyse in detail the predictions of the model for several possible signatures. In addition to adopting two different parametrizations for the PES, the calculations are performed for various assumptions on critical ingredients of the theory which modelling is either uncertain or debated. This will permit to probe the robustness of the conclusion regarding the sole influence of the PES.

\section{Fission of heavy systems}

For the reasons outlined above, we start the discussion with the heavy ${ }^{260} \mathrm{Rf}$ system. The ability of the present code, using the FRLDM prescription and assuming a reduced one-body dissipation, to reproduce the experimental data available [59] was already noticed in Ref. [27]. 


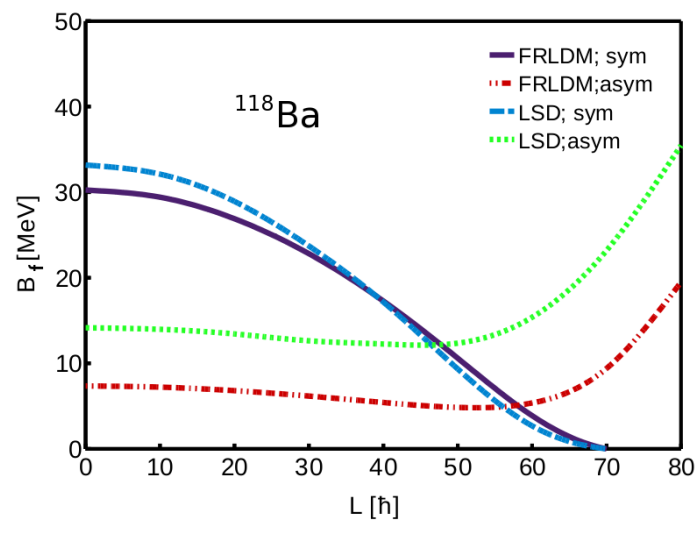

FIG. 4: (colour on-line) Evolution of the fission barrier with $L$ for the ${ }^{118} \mathrm{Ba}$ compound nucleus: symmetric (full violet) and asymmetric (double dashed-dotted red) barriers calculated with FRLDM; symmetric (dashed blue) and asymmetric (dotted green) barriers calculated with LSD.

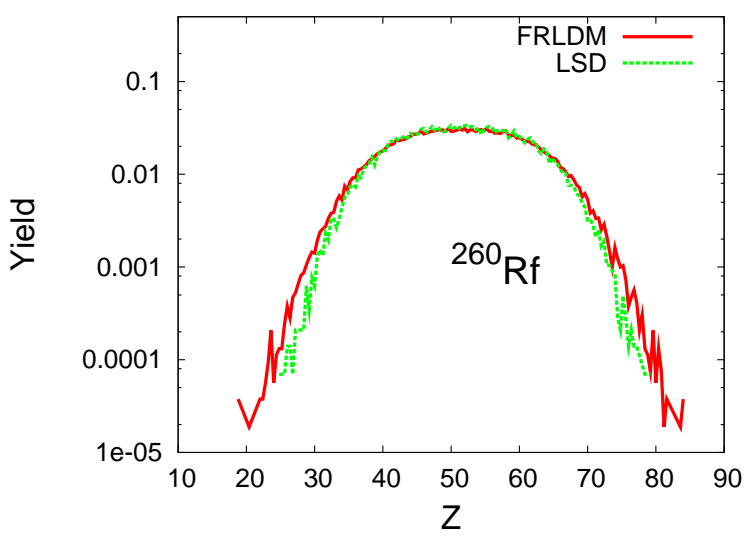

FIG. 5: (colour on-line) Charge yield distribution of the fission fragments produced in the reaction ${ }^{20} \mathrm{Ne}(142 \mathrm{MeV})+{ }^{240} \mathrm{Pu} \rightarrow{ }^{260} \mathrm{Rf}$. The full red (dashed green) line depicts the result of the calculation utilizing the FRLDM (LSD) potential. The level-density parameter is set to $a=A / 10 \mathrm{MeV}^{-1}$ and friction is reduced according to $k_{s}=0.5$.

Fission of medium-mass nuclei

The calculations which we performed show little sensitivity to whether FRLDM or LSD is used for the PES. The fission-fragment $Z$ distributions computed with the two PES parametrizations are compared in Fig. 5. The outcome of the calculation in terms of asymmetry distribution is converted into mass and charge distributions as described in Ref. [27]. A constant level-density parameter $a=A / 10 \mathrm{MeV}^{-1}$ and a friction reduction factor $k_{s}=0.5$ are assumed. The $Z$ distributions for fission of ${ }^{260} \mathrm{Rf}$ are observed to be very similar, being slightly broader for FRLDM due to the softer energy landscape in this case. The corresponding $A$ and TKE distributions are found nearly independent of the PES formula as well, and in good agreement with the experimental data [59]. The pre-scission neutron multiplicities obtained with the two PES agree within $\sim 1 \%$, and also compare well with the measured value. The good reproduction of the data leads confidence into the reliability of the present calculations for modelling fission dynamics. We note that a similar agreement between theory and experiment was achieved for ${ }^{260} \mathrm{Rf}$ by Karpov et al. [27] with a smaller value of the friction reduction factor, namely $k_{s}=0.2$. The difference in the $k_{s}$ values adopted in Ref. [27] and here is related to the use of slightly different parameters of the statistical model. The difference remains nonetheless within the range of uncertainty observed for $k_{s}$ by Karpov et al.. The present survey of the ${ }^{260} \mathrm{Rf}$ system corroborates the expected limited sensitivity of fission of heavy systems to the details of the PES, and namely the moderate influence of curvatures effects. Fission of heavy nuclei seems thus not be best suited for probing the details of the potential energy surface and its parametrization.
For fission of the neutron-deficient Barium, the sensitivity to the PES prescription is found to markedly differ from that observed in the heavy-mass region. Since the angular momentum plays an important role in fission of intermediate-fissility systems, the $L$ distribution of the initial ${ }^{118} \mathrm{Ba}$ compound nucleus produced in the ${ }^{78} \mathrm{Kr}(429 \mathrm{MeV})+{ }^{40} \mathrm{Ca}$ collision is shown in Fig. 6. Events leading to the formation of an evaporation residue and events ending up with fission are considered separately. In the calculations of the figure, the magnitude of onebody dissipation is reduced according to $k_{s}=0.2$, and two hypothesis are assumed for the level-density parameter $a$. For a given value of the latter $\left(a=A / 8 \mathrm{MeV}^{-1}\right.$ in Fig. 6) the weight of the smallest $L$ values contributing to fission is larger with LSD as compared to FRLDM. This difference in the partition of the angular momentum between the evaporation and fission channels can be cancelled out by assuming a different level-density parameter for the two PES. That is illustrated in Fig. 6 with a calculation performed with $a=A / 12 \mathrm{MeV}^{-1}$ in combination with the LSD potential. It shows that the $L$ distribution does not stand for a relevant signature of the PES parametrization. In other words, within the uncertainty inherent to model ingredients, it is not possible, on the basis of the spin distribution alone, to disentangle between the FRLDM and LSD prescriptions. Note that the $L$ observable, extracted experimentally from measurements of $\gamma$-ray multiplicities, may nonetheless be pertinent for studying other inputs of the theory [66].

In Section III A we conjectured the possible occurrence of a Businaro-Gallone-like transition in the fission of ${ }^{118} \mathrm{Ba}$ around $L \sim(40-60) \hbar$. From the angular mo- 
mentum distributions of fission events displayed in Fig. 6 , there is every indication that this transition may be difficult to observe. Due to the moderate fissility of the system, only the highest partial waves contribute to fission, and the fission yield is predicted to die down precisely for those $L$ values where the asymmetric and symmetric barriers become similar, see Fig. 4, i.e. at the location of the Businaro-Gallone transition. A hint for the presence of such a transition will nonetheless be discussed later below.

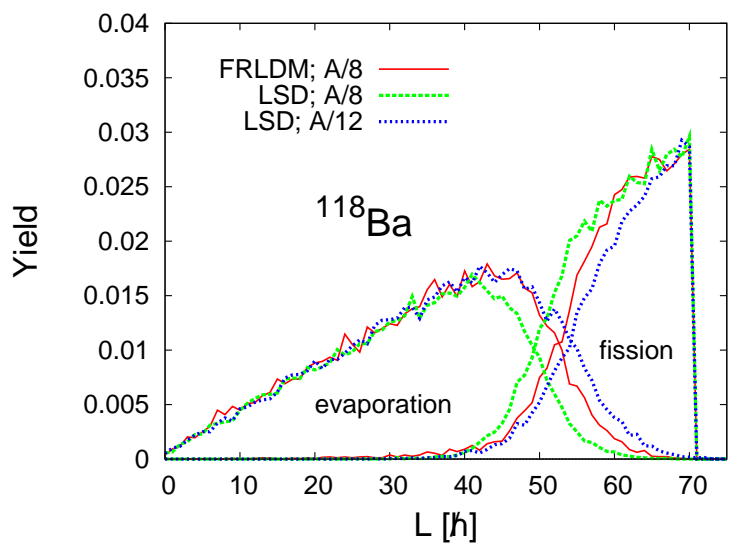

FIG. 6: (colour on-line) Angular momentum distribution of initially excited ${ }^{118} \mathrm{Ba}$ nuclei produced in the reaction ${ }^{78} \mathrm{Kr}(429 \mathrm{MeV})+{ }^{40} \mathrm{Ca}$ for evaporation-residue and fission events. The thin full red (thick dashed green) line depicts the result of the calculation assuming a PES derived from the FRLDM (LSD) potential with the level-density parameter $a=A / 8 \mathrm{MeV}^{-1}$. The dashed blue line shows the prediction computed with the LSD prescription and $a=A / 12 \mathrm{MeV}^{-1}$. In all calculations, $k_{s}$ is set to 0.2 .

The outcome of a set of calculations for commonly investigated fission observables is summarized in Table I. The fission probability $P_{f}$, the mean neutron $\left\langle n_{\text {pre }}\right\rangle$, proton $\left\langle p_{\text {pre }}\right\rangle$, and $\alpha$-particle $\left\langle\alpha_{\text {pre }}\right\rangle$ pre-scission multiplicities, the variance $\sigma_{A}^{2}\left(\sigma_{Z}^{2}\right)$ of the fission-fragment $A(Z)$ distribution, the mean value $\langle T K E\rangle$ and variance $\sigma_{T K E}^{2}$ of the total kinetic energy distribution as well as the mean temperature $\left\langle T_{s c}\right\rangle$ at scission are given. Mean fissionfragment masses (charges) are not indicated since the calculated distributions are in all cases dominated by symmetric fission and peak at about half of the mass (charge) of the ${ }^{118} \mathrm{Ba}$ compound. Mass and charge variances have been extracted from a limited range of $Z$ values around symmetry $(5 \leq Z \leq 47)$ in order to exclude potential asymmetric wings and statistical fluctuations. Also given in the table are the mean particle multiplicities $\left(\left\langle n_{\text {eva }}\right\rangle,\left\langle p_{\text {eva }}\right\rangle,\left\langle\alpha_{\text {eva }}\right\rangle\right)$ associated with the formation of an evaporation residue.

Various combinations of the PES prescription and the level-density parameter are investigated in Table I while $k_{s}$ is set to 0.2 in all calculations. Besides a constant value $(a=A / x)$ and the formula of Ignatyuk [50], the parametrization of the level density by Pomorski et al. [67] is considered together with the LSD potential. Both the Ignatyuk and Pomorski formulae include the deformation dependence of the level density accounting for surface effects. The prescription by Pomorski contains, in addition, a deformation-dependent curvature term, and is therefore consistent to be used in combination with the LSD parametrization. Note that, while for $a=A / x$ the ratio of the level-density parameter at the saddle point to that at the ground state $\left(a_{f} / a_{n}\right)$ is strictly unity, it generally differs from 1 for Ignatyuk and Pomorski prescriptions. This ratio is known to be among the critical ingredients of any statistical and dynamical model of fission (see e.g. Refs. [68-70] and therein). Attention will therefore be paid on its uncertainty along the discussion of PES effects.

The survey of Table I shows that for decaying excited ${ }^{118} \mathrm{Ba}$ compound nuclei, among all observables considered here, the fission-fragment $A, Z$ and TKE distributions are particularly sensitive to the parametrization of the PES. On the contrary, for a given level-density prescription, the pre-scission particle multiplicities and temperature at scission do almost not depend on the PES prescription. These quantities are seen to be primarily governed by the assumption used for the level density [71]. As far as the fission probability is concerned, the influence of the level-density parameter appears to be comparable in magnitude to the influence of the PES $[68,71]$. The larger $P_{f}$ predicted with the LSD model is mostly due to the broader range of angular momentum covered by fission for a given level-density parameter, see Fig. 6. When used with another level density ( $a=A / 12 \mathrm{MeV}^{-1}$ in the table) which corresponds to a similar $L$ window, the LSD prescription yields a fission probability close to that calculated with FRLDM for $a=A / 8 \mathrm{MeV}^{-1}$. Note that the results obtained with Ignatyuk's level-density prescription are similar to those computed with $a=A / 12 \mathrm{MeV}^{-1}$ : Neglecting deformation effects in the formula of Ref. [50] gives a parameter a close to $A / 12 \mathrm{MeV}^{-1}$ for ${ }^{118} \mathrm{Ba}$. A completely different dependence on the model ingredients is observed for the mass, charge and total kinetic energy distributions. The quantities $\sigma_{A}^{2}, \sigma_{Z}^{2},\langle T K E\rangle$ and $\sigma_{T K E}^{2}$ are found to strongly depend on the PES prescription. In particular, the variances are much larger when the FRLDM parametrization is used, while the mean total kinetic energy is slightly smaller. The narrower $A$ and $Z$ distributions obtained with the LSD model are due to the stiffer landscape at, and beyond, the saddle point, see Fig. 3. According to the close connection between the Coulomb repulsion and the total kinetic energy of the fission fragments at scission [27], the smaller $\langle T K E\rangle$ computed with FRLDM is to be correlated to the smaller width of the mass and charge distributions. As obvious from the variances and detailed below, the FRLDM leads to a larger amount of asymmetric fission partitions, which are characterized by lower TKE's.

The calculated fission-fragment charge distribution is 
TABLE I: Results of the calculation for various observables and various combinations of the PES and the level-density prescription for the decay of excited ${ }^{118} \mathrm{Ba}$ compound nuclei produced in the reaction ${ }^{78} \mathrm{Kr}(429 \mathrm{MeV})+{ }^{40} \mathrm{Ca}$. In all calculations presented here $k_{s}$ is set to 0.2 . ( $\langle T K E\rangle$ and $\left\langle T_{s c}\right\rangle$ are given in $[\mathrm{MeV}], \sigma_{E_{k}}^{2}$ is given in $\left.\left[\mathrm{MeV}^{2}\right]\right)$

\begin{tabular}{|c|c|c|c|c|c|c|c|}
\hline \multirow[b]{2}{*}{$a$} & \multicolumn{3}{|c|}{ FRLDM } & \multicolumn{4}{|c|}{ LSD } \\
\hline & $\mathrm{A} / 8$ & $\mathrm{~A} / 12$ & {$[50]$} & $\mathrm{A} / 8$ & $\mathrm{~A} / 12$ & {$[50]$} & {$[67]$} \\
\hline$P_{f}$ & 0.42 & 0.31 & 0.34 & 0.49 & 0.39 & 0.43 & 0.57 \\
\hline$\left\langle n_{\text {pre }}\right\rangle$ & 0.03 & 0.11 & 0.07 & 0.03 & 0.09 & 0.07 & 0.02 \\
\hline$\left\langle p_{\text {pre }}\right\rangle$ & 0.06 & 0.23 & 0.15 & 0.04 & 0.17 & 0.11 & 0.03 \\
\hline$\left\langle\alpha_{\text {pre }}\right\rangle$ & 0.03 & 0.15 & 0.10 & 0.03 & 0.13 & 0.08 & 0.02 \\
\hline$\left\langle n_{\text {eva }}\right\rangle$ & 1.31 & 0.99 & 1.08 & 1.38 & 1.04 & 1.14 & 1.33 \\
\hline$\left\langle p_{\text {eva }}\right\rangle$ & 3.00 & 2.16 & 2.38 & 3.09 & 2.26 & 2.49 & 2.85 \\
\hline$\left\langle\alpha_{e v a}\right\rangle$ & 1.47 & 1.65 & 1.65 & 1.43 & 1.62 & 1.63 & 1.56 \\
\hline$\sigma_{A}^{2}$ & 371.86 & 454.86 & 434.90 & 126.13 & 158.81 & 153.97 & 152.08 \\
\hline$\sigma_{Z}^{2}$ & 83.82 & 102.42 & 98.05 & 28.69 & 35.98 & 34.91 & 34.50 \\
\hline$\langle T K E\rangle$ & 87.48 & 85.89 & 86.25 & 94.17 & 94.04 & 94.10 & 93.73 \\
\hline$\sigma_{E_{k}}^{2}$ & 197.13 & 248.91 & 234.65 & 35.83 & 51.18 & 47.80 & 50.44 \\
\hline$\left\langle T_{s c}\right\rangle$ & 1.67 & 1.99 & 1.91 & 1.68 & 2.02 & 1.92 & 1.90 \\
\hline
\end{tabular}

displayed in Fig. 7 for various combinations of the model ingredients. The FRLDM (LSD) parametrization is considered in the top (bottom) panel. Absolute cross sections were obtained after normalizing the fission yields to the calculated fusion cross section [25]. Different lines refer to various formulations of the level-density parameter. As inferred above from the analysis of the variances, the $Z$ distribution strongly depends on the prescription used for the PES. It is well established [68, 69, 71, 72] that different combinations of the level density and the fission barrier can lead to similar predictions, due to the opposite influence of the $a_{f} / a_{n}$ and $B_{f}$ parameters. Note that the fission barrier is a property of the potential energy surface i.e. it depends on the PES prescription. This anti-correlation between the level density and the PES is behind the observation made along the discussion of Fig. 6. It is obvious from Fig. 7 that the conclusion on the influence of the potential energy surface holds whatever parametrization is used for the level density. In other words, for fissioning ${ }^{118} \mathrm{Ba}$ nuclei, the large difference between the results computed with the FRLDM and LSD potentials can not be cancelled out by a different mod- elling of the level density. The influence of the latter on the $Z$ distribution is found much smaller in magnitude than the one related to the PES prescription. The same is true for the mass distribution.

The large discrepancy between the charge distributions cannot be explained neither by a difference in the angular momenta. As observed in Fig. 6, the $L$ ranges can be matched by different choices of the level density, with a limited modification on the $Z$ distribution, see Fig. 7 . Gontchar et al. [31] also observed that $L$ effects are not dominant in determining the fission partition. From a compilation of a huge amount of data, Rusanov et al. [60] concluded that the width of the fission-fragment mass (charge) distribution is primarily governed by the temperature $T$ and the stiffness $d^{2} V / d \alpha^{2}$ of the potential energy surface with respect to the asymmetry coordinate. Table I shows that $\left\langle T_{s c}\right\rangle$ is nearly independent on the parametrization of the PES, and more strongly depends on $a$. Our result therefore suggests that the difference observed in Fig. 7 between the $Z$ distributions for the fission of ${ }^{118} \mathrm{Ba}$ is fully to be attributed to the difference in the stiffness between the two PES models, and more generally in the overall FRLDM and LSD potential energy topology. (We note that the location of the relevant point along the trajectory at which $T$ and $d^{2} V / d \alpha^{2}$ have to be evaluated to calculate $\sigma_{A}^{2}$ is still debated. Some works favor the saddle point, whereas others give evidence for the decisive influence of the scission point, and some others for a point located somewhere in between (see Ref. [60] and therein). For the present system, the saddle and scission points are very close, and so are the corresponding temperatures and stiffnesses.)

The fission-fragment $Z$ distribution calculated with the FRLDM parametrization clearly exhibits asymmetric wings. These are to be connected with the development of asymmetric valleys in the corresponding PES, see Fig. 3 (top). For the LSD potential energy prescription, side-peaks are much smaller. This can only partly be explained by the higher separation ridge between the symmetric and asymmetric valleys in this case, see Fig. 3 (bottom). The weaker magnitude of asymmetric splits with LSD is more likely to be related to the dependence of the asymmetric and symmetric fission barriers on spin, see Fig. 4. For FRLDM the asymmetric barrier is smaller than the symmetric one up to $L=58 \hbar$, whereas it drops below the symmetric barrier already at $L=48 \hbar$ with LSD. According to Fig. 6 fission sets in from $L \sim 40 \hbar$ on, independently of the choice of the PES prescription. As a consequence, the range of angular momentum within which asymmetric fission favorably competes with symmetric fission is wider for FRLDM. We emphasize that, although the presence of asymmetric fission is undeniable, its contribution to the total fission cross section is very weak for both PES (note the logarithmic scale of the vertical axis in the figure).

The correlation between the fission-fragment mass and total kinetic energy is considered in Fig. 8. Around symmetry, a triangular-like shape typical of fission of an 

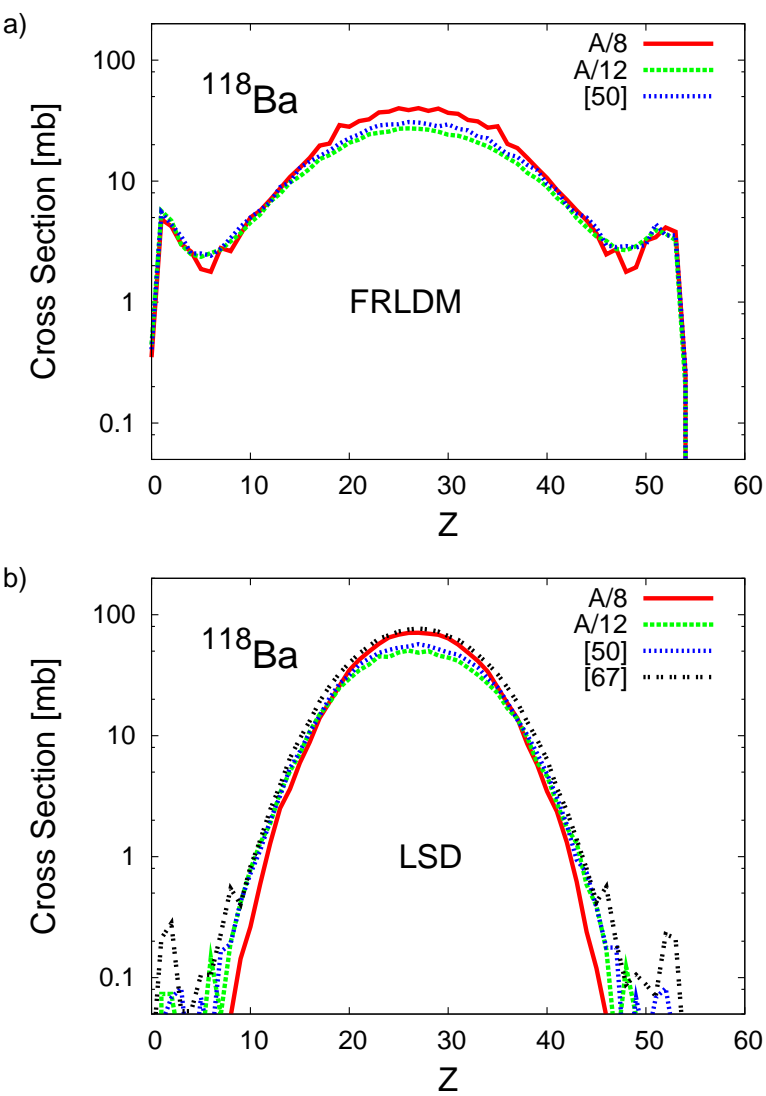

FIG. 7: (colour on-line) Charge distribution of the fission fragments produced in the reaction ${ }^{78} \mathrm{Kr}(429 \mathrm{MeV})+{ }^{40} \mathrm{Ca} \rightarrow$ ${ }^{118} \mathrm{Ba}$. The top (bottom) panel shows the calculation performed with the FRLDM (LSD) potential. The various lines refer to different parametrizations of the level density as indicated in the right corner of the figures. In all calculations, $k_{s}$ is set to 0.2 .

equilibrated excited compound nucleus is observed [27] for the two PES parametrizations. These correlations show that the larger $\sigma_{E_{k}}^{2}$ variance predicted by FRLDM as compared to LSD is due to the larger amount of asymmetric fission partitions with this potential energy prescription.

While discussing Fig. 4 we mentioned the possibility of observing a Businaro-Gallone-like transition with increasing spin for the fission of ${ }^{118} \mathrm{Ba}$ nuclei. From the angular momentum distribution presented in Fig. 6 we concluded that such a bifurcation might be difficult to evidence in experiment, because the nuclei with those partial waves expected to lead to asymmetric fission have a small fission probability due to the high absolute value of $B_{f}$ at moderate $L$. The contribution to fission of events with $L$ below $\sim 48 \hbar$ and $\sim 58 \hbar$ for LSD and FRLDM, respectively, is finally found to lead to a small amount of asymmetric fission partitions as seen in Fig. 7. Note that the latter figure integrates the whole $L$ range contributing to fission up to $L_{c r i t}$ i.e. the highest partial waves dominate. Probing the aforementioned Businaro-Gallone-like transition requires sampling the $L$ distribution. We performed a detailed analysis of the fission-fragment $Z$ distribution for different selections on the range of $L$ 's contributing to fission. Although the statistics is quite low at low spin, the asymmetric component is found to clearly grow with decreasing angular momentum.

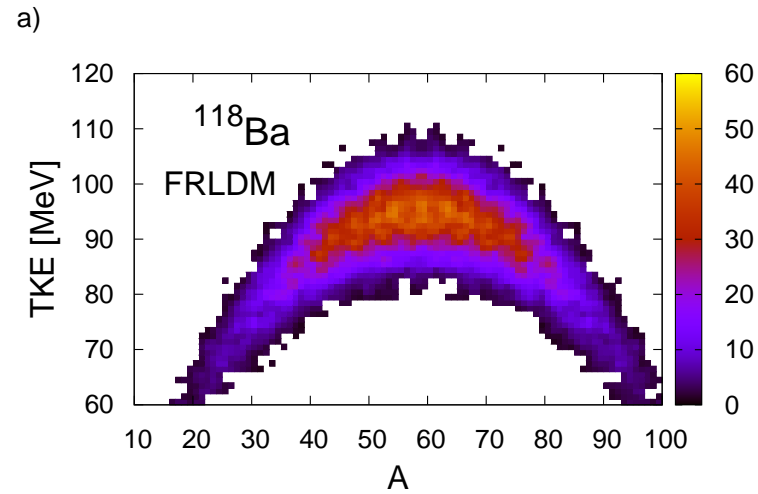

b)

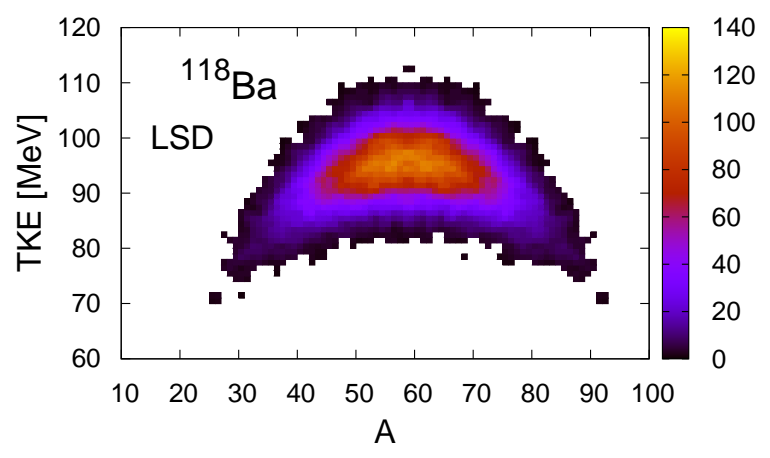

FIG. 8: (colour on-line) Correlation between the mass $A$ and total kinetic energy TKE of the fission fragments produced in the reaction ${ }^{78} \mathrm{Kr}(429 \mathrm{MeV})+{ }^{40} \mathrm{Ca} \rightarrow{ }^{118} \mathrm{Ba}$. The top (bottom) panel shows the calculation performed with the FRLDM (LSD) potential. In all calculations, the level-density parameter is $a=A / 8 \mathrm{MeV}^{-1}$ and $k_{s}$ is set to 0.2 .

In addition to the examination of the influence of the level-density parameter, we investigated the robustness of our results vis a vis the uncertainty on the magnitude of friction. Table II compares the predictions of a set of calculations obtained with $k_{s}=0.2$ and $k_{s}=1.0$ for the two PES prescriptions. Comments similar to those drawn about Table I apply. While friction largely governs the particle pre-scission multiplicities, the fission probability results from the complex interplay between $k_{s}$ and PES effects. At the same time, the strong dependence of the fission-fragment $A, Z$ and TKE 
distribution widths is impossible to be counterbalanced by a variation in the magnitude of nuclear dissipation. As a further control of the possible influence of simplistic model parameters, we investigated the sensitivity of the results on the modelling of particle evaporation, and obtained that the above conclusions regarding the PES influence are left un-changed.

TABLE II: Results of the calculation for various observables and various combinations of the PES and the friction reduction factor (see the text) for the decay of excited ${ }^{118} \mathrm{Ba}$ compound nuclei produced in the reaction ${ }^{78} \mathrm{Kr}(429 \mathrm{MeV})+{ }^{40} \mathrm{Ca}$. In all calculations presented here $a$ is set to $A / 8 \mathrm{MeV}^{-1}$. $\left(\langle T K E\rangle\right.$ and $\left\langle T_{s c}\right\rangle$ are given in $[\mathrm{MeV}], \sigma_{E_{k}}^{2}$ is given in $\left.\left[\mathrm{MeV}^{2}\right]\right)$

\begin{tabular}{ccccc}
\hline \hline \multicolumn{4}{c}{ FRLDM } & \multicolumn{2}{c}{ LSD } \\
\hline$k_{s}$ & 0.2 & 1.0 & 0.2 & 1.0 \\
& & & & \\
\hline & & & & \\
$P_{f}$ & 0.42 & 0.33 & 0.49 & 0.41 \\
& & & & \\
$\left\langle n_{\text {pre }}\right\rangle$ & 0.03 & 0.05 & 0.03 & 0.05 \\
$\left\langle p_{\text {pre }}\right\rangle$ & 0.06 & 0.13 & 0.04 & 0.09 \\
$\left\langle\alpha_{\text {pre }}\right\rangle$ & 0.03 & 0.07 & 0.03 & 0.06 \\
$\sigma_{A}^{2}$ & 371.86 & 365.82 & 126.13 & 119.90 \\
$\sigma_{Z}^{2}$ & 83.82 & 82.56 & 28.69 & 27.21 \\
& & & & \\
$\langle T K E\rangle$ & 87.48 & 85.46 & 94.17 & 93.61 \\
$\sigma_{E_{k}}^{2}$ & 197.13 & 193.17 & 35.83 & 37.21 \\
$\left\langle T_{s c}\right\rangle$ & 1.673 & 1.638 & 1.676 & 1.636 \\
& & & & \\
\hline \hline
\end{tabular}

Comparison with previous work

In Ref. [31] Gontchar et al. used two-dimensional Langevin calculations to investigate in detail the influence of several model ingredients on the dynamical evolution of heavy fissioning systems. In particular, they compared the widths of the fission-fragment mass distribution obtained with either the RLDM [16] or the FRLDM $[22,23]$ prescriptions. For compound nuclei with $A \geq 200$, they obtained a strong dependence of $\sigma_{A}^{2}$ on the PES parametrization. The different behaviours observed by Gontchar et al. and in the present work for heavy systems is understood as follows. The RLDM and FRLDM parametrizations which are confronted in Ref. [31] yield fission barriers which more sizeably differ from each other than the FRLDM and LSD barri- ers do $[17,23]$. This larger difference, combined to the higher fissility of the systems studied in Ref. [31], affects the temperature at, and beyond, the saddle point (see Fig. 13 of Ref. [31]), and finally the width of the mass distribution. Provided the PES prescriptions are different enough, the influence of the temperature and of the stiffness of the PES can thus become of similar magnitude, and heavy systems may be well suited for comparing RLDM and FRLDM. We note also that Gontchar et al. restricted to $L=0 \hbar$ for which the difference in the barriers between RLDM and FRLDM is largest. This limitation magnifies the effect. The present work focuses on finer details of the PES topology, which unambiguous evidence requires the study of lighter systems. For a medium-mass compound nucleus we obtained similar $B_{f}$ values with FRLDM and LSD for those partial waves which have a strong contribution to fission. Furthermore, we observed nearly identical temperatures at scission. In spite of this, the fission-fragment $A$ and $Z$ distributions differ sizeably. We understand this result as a strong sensitivity to the sole influence of the stiffness of the PES (see also discussion above). The present study complements the work of Gontchar et al. [31] done in the heavymass region. In addition, it permits going a step further by (i) the introduction, for the first time in such calculations, of the new LSD potential which has shown superior in describing experimental masses, (ii) the investigation of a medium-mass system, and (iii) the search of selectively sensitive signatures of subtle details of the PES which are difficult to pin down indisputably in highly fissile nuclei.

In Ref. [48, 73] Ryabov et al. performed a detailed analysis of the evolution of the widths of the fission-fragment $A$ and TKE distributions as a function of angular momentum over a wide mass range from ${ }^{162} \mathrm{Yb}$ up to ${ }^{244} \mathrm{Cm}$. The temperature-dependent FRLDM parametrization was assumed for computing the potential energy. Ryabov et al. observed that the dependence of $\sigma_{A}^{2}$ on spin is directly connected to the dependence of pre-scission evaporation on spin, i.e. the role of $T_{s c}$ dominates over that played by the PES stiffness in determining the evolution of the shape of the mass distribution with increasing angular momentum. However, they noted that the magnitude of $\sigma_{A}^{2}$ is mostly determined by the potential energy landscape. For a less fissile system, the present work corroborates the findings of $[48,73]$ restricted to a particular PES. By considering two different PES pescriptions, we additionally show that the absolute value of $\sigma_{A}^{2}$ (integrated over all $L$ 's) critically depends on the PES parametrization in the medium-mass region, while the role of $T_{s c}$ is minor. Furthermore, while sampling the results as a function of $L$ (see above), and for a given PES formula, we observe the dependence of $\sigma_{A}^{2}$ on $L$ foreseen by Ryabov et al:: Namely, the mass distribution gets broader with decreasing spin for fission of ${ }^{118} \mathrm{Ba}\left(Z^{2} / A=26.6\right)$. 


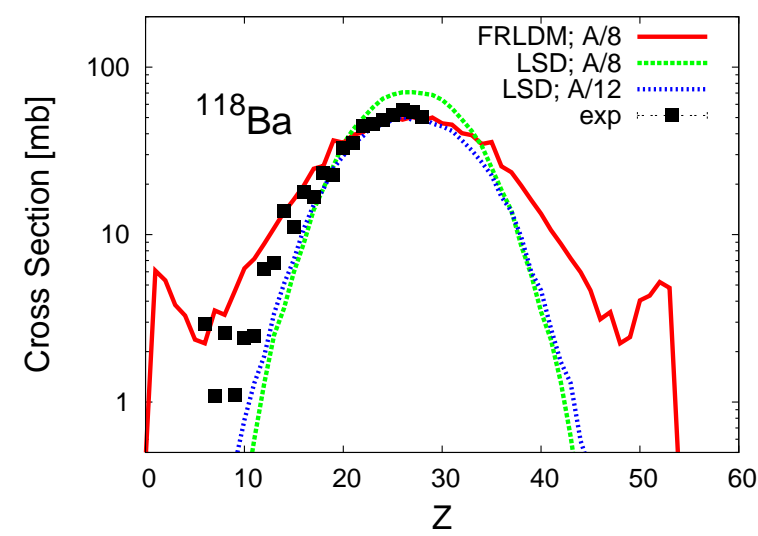

FIG. 9: (colour on-line) Charge distribution of the fission fragments produced in the reaction ${ }^{78} \mathrm{Kr}(429 \mathrm{MeV})+{ }^{40} \mathrm{Ca} \rightarrow$ ${ }^{118} \mathrm{Ba}$. The various lines refer to different combinations of the PES and level-density parametrizations as indicated in the right corner of the figure. In all calculations, $k_{s}$ is set to 0.2 . The experimental points measured by Ademard et al. [58] are shown as solid squares. Error bars are smaller than the symbols.

\section{Comparison with experimental data}

The reaction ${ }^{78} \mathrm{Kr}(429 \mathrm{MeV})+{ }^{40} \mathrm{Ca} \rightarrow{ }^{118} \mathrm{Ba}$ was recently studied at the Grand Accélérateur National d'Ions Lourds (GANIL), Caen, and the fragment production cross section was measured over a wide range [58]. The experimental elemental cross sections are superimposed to some of the model calculations in Fig. 9. Three theoretical distributions borrowed from Fig. 7 are shown in order to pin down the large influence of the PES, on one side, and the smaller influence of the uncertainty in the level-density parameter, on the other side. In the symmetric region $(Z \geq 10)$, experimental and calculated cross sections agree within a factor of $\sim 5$. The staggering in the data for $Z \leq 10$ is due to even-odd effects which modelling is out of the scope of the present code. The mean $\langle T K E\rangle$ value obtained with the FRLDM and LSD prescriptions, which are close to each other (see Table I), are in reasonable agreement with experiment [58] and Viola systematics [74]. The comparison between the experimental and theoretical $Z$ distributions is to be considered as purely qualitative. None of the theoretical curves perfectly matches the data and, although possible, no attempt was done to improve on this point. Doing so, would in addition have little sense since Ademard et al. [58] found a sizeable contribution from quasi-fission in the measured fragment production. The present model is intended to describe fission of a fully equilibrated compound nucleus, and is not suited for quasi-fission events. The latter mechanism is known to yield a broader fission-fragment $A(Z)$ distribution as compared to fusion-induced fission (see Ref. [75] and therein). Hence, in the presence of a large quasi-fission component, the experimental distribution is expected larger than what predicted with a model restricted to compound-nucleus fission. The comparison presented in Fig. 9 may therefore be interpreted as speaking in favor of the LSD potential energy surface as most suited. However, at this stage, this remark remains purely speculative, and further studies are required to settle the point. The qualitative confrontation to the experimental data is nonetheless sufficient to confirm the relevance of fissionfragment elemental production cross sections to investigate the details of the modelling of the PES in mediummass nuclei.

As another test of the present calculations, we consider experimental data on neutron multiplicities that exist for a nearby system. In Ref. [76] excited ${ }^{126} \mathrm{Ba}$ compound nuclei were produced at an excitation energy of $\sim 132 \mathrm{MeV}$ with a projectile/target combination for which quasi-fission is expected to be weak. The multiplicities predicted by the present code for this system amount to $\left\langle n_{\text {pre }}\right\rangle^{F R L D M}=2.42$ and $\left\langle n_{\text {pre }}\right\rangle^{L S D}=2.65$ for the FRLDM and LSD models, respectively. These values are in very good agreement with the measurement which yielded $\left\langle n_{\text {pre }}\right\rangle^{\exp }=2.52 \pm 0.12$ [76]. Together with the good description of the ${ }^{260} \mathrm{Rf}$ data noticed above, this result leads further confidence into the power of the code used here, and the reliability of our conclusions regarding PES effects. In addition, it shows again the weak sensitivity of the $\left\langle n_{p r e}\right\rangle$ observable to the potential energy parametrization. Previous one-dimensional calculations $[33,36]$ for the ${ }^{126} \mathrm{Ba}$ system focused on the RLDM and LSD approaches, which respectively gave $\left\langle n_{\text {pre }}\right\rangle^{R L D M}=1.5$ and $\left\langle n_{\text {pre }}\right\rangle^{L S D}=2.48$. That was already interpreted as the evidence of strong curvature effects for medium-mass nuclei. As compared to the present work, these calculations neglected the congruence term in the potential energy, while the deformation dependence of the particle transmission coefficients were accounted for as in Ref. [36, 77]. As another aspect of the model which would be relevant to improve on, namely for fission of light systems, we shall mention the flexibility of the nuclear shape parametrization [78].

The FRLDM potential energy prescription is used in most of the modern dynamical Langevin calculations, while the LSD parametrization was investigated for the first time in the present work in combination with a highly realistic multi-dimensional code. The comparison with the available experimental data shows that, for a medium-mass nucleus and the relevant observable, the predictions by FRLDM and LSD lie on both side of the measurement. It furthermore demonstrates that, in this mass region, PES effects can be of similar magnitude than the influence of a mixture of different reaction mechanisms (quasi- and fusion-fission presently) on the fission-fragment distribution can be. This observation emphasizes the importance of looking into the issue of the best suited parametrization of the PES. While most work done during the last two decades concentrated on 
model ingredients such as nuclear viscosity, level densities and particle emission barriers, the present work highlights that, depending on the system, the choice of the parametrization of the potential energy landscape may influence the conclusion drawn about other nuclear properties extracted from the calculation.

\section{CONCLUSION}

A comprehensive study of the influence of the potential energy surface on the dynamical evolution of hot and rotating fissioning nuclei was performed. The importance of curvature effects was investigated using two parametrizations of the macroscopic potential energy i.e. the Finite Range Liquid Drop Model and the LublinStrasbourg Drop prescription. According to the puzzling interplay between static and dynamical effects, realistic calculations were performed by using a state-of-art three dimensional Langevin code. Various combinations of the model ingredients were assumed in order to unambiguously reveal effects related solely to the topology of the PES. In particular, the dependence of the conclusion regarding the PES on the uncertainty inherent to the leveldensity parameter and the friction strength was checked into thoroughly.

While the outcome of the dynamical calculations for a very heavy system are found nearly independent on the PES prescription used, it is observed that the predictions for fission of the intermediate-fissility ${ }^{118} \mathrm{Ba}$ nucleus can strongly depend on the parametrization of the potential energy. The present work also reveals that the sensitivity to PES effects clearly relies on the choice of the observables. In particular, we observe that the characteristics of the fission-fragment mass, charge and total kinetic energy distributions noticeably differ depending on the PES pre- scription. The leading term in determining these observables is the stiffness of the potential energy landscape, whereas the fission probability and pre-scission particle multiplicities depend as well on (or are even primarily governed by) the level-density parameter and the magnitude of nuclear viscosity.

The comparison of the predictions for the FRLDM and LSD prescriptions as obtained with a realistic model in a multi-dimensional deformation space and under identical conditions points to the importance of the choice of the potential energy surface. The latter may influence the conclusions drawn on nuclear properties extracted from the calculation. In addition, by highlighting the dependence of the sensitivity of the observable on the size of the system, the present work defines what are the experimental conditions required to carefully investigate the potential energy landscape. Nuclei with intermediate fissility are observed to be well suited to probe thoroughly the PES topology and its parametrization in minute detail. The study of medium-mass systems shall thus permit to improve further the description of the macroscopic potential. These developments may benefit throughout the whole nuclear chart.

\section{Acknowledgments}

This work has been supported by the ANR (Agence Nationale pour la Recherche) PROVA No.7303 and the COPIN-IN2P3 Polish-French Collaboration under Contract No.09-136. One of us (P.N.N.) is grateful to the Grand Accélérateur National d'Ions Lourds (GANIL) for hospitality and financing of his stay at the laboratory. We are very thankful to Prof. G.D. Adeev, Prof. K. Pomorski and Dr. J. Bartel for enlightening discussions which permitted to improve the quality of the work. Prof. K. Pomorski and D. Boilley are acknowledged for critical reading of the manuscript.
[1] S. Aberg, H. Flocard, W. Nazarewicz, Ann. Rep. Nucl. Part. Sci. 40, 439 (1990).

[2] J.L. Egido, L.M. Robledo, R.R. Chasman, Phys. Lett. B 393, 13 (1997).

[3] J.F. Berger, M. Girod, D. Gogny, Nucl. Phys. A 502, 82c (1989).

[4] P. Möller and J.R. Nix, At. Data Nucl. Data Tables 39, 213 (1988).

[5] P. Möller, A.J. Sierk, R. Bengtsson, H. Sagawa, T. Ichikawa, Phys. Rev. Let. 103 , 212501 (2009).

[6] P. Möller, A.J. Sierk, A. Iwamoto, Nature London 409, 585 (2001).

[7] J. Dudek, K. Pomorski, N. Schunck, N. Dubray, European Phys. J. A 20, 165 (2004).

[8] A. Baran, Z. Łojewski, K. Sieja, M. Kowal, Phys. Rev. C 72, 044310 (2005).

[9] A. Maj,et al., Nucl. Phys. A 731, 319c (2004).

[10] V.M. Strutinsky, Nucl. Phys. A 95, 420 (1967), A 122, 1 (1968).

[11] H.A. Bethe and R.F. Bacher, Rev. Mod. Phys. 8, 82
(1936).

[12] C. Weizsäcker, Z. Phys. 96, 431 (1935).

[13] W.D. Myers and W.J. Świątecki, Nucl. Phys. 81, 1 (1966).

[14] L. Meitner and O.R. Frisch, Nature 143, 239 (1939).

[15] N. Bohr and J.A. Wheeler, Phys. Rev. 56, 426 (1939).boru

[16] S. Cohen, F. Plasil, W.J. Świątecki, Ann. Phys. 82, 557 (1974).

[17] K. Pomorski, J. Dudek, Phys. Rev. C 67, 044316 (2003).

[18] D.L. Hill and J.A. Wheeler, Phys. Rev. 89, 1102 (1953).

[19] H.V. Groote and E.. Hilf, Nucl. Phys. A 129, 513 (1969).

[20] M.S. Anthony, Nuclide Chart 2002, Strasbourg, 2002, Impression Francois, Haguenau, France.

[21] K. Pomorski, F. Ivanyuk, Int. J. Mod. Phys. E 18, 900 (2009)

[22] A.J. Sierk, Phys. Rev. C 33, 2039 (1986).boru

[23] H.J. Krappe, J.R. Nix, and A.J. Sierk, Phys. Rev. C 20, 992 (1979).

[24] P. Möller, A.J. Sierk, A. Iwamoto, Phys. Rev. Lett. 92, 
072501 (2004).

[25] P. Froebrich, I.I. Gontchar, Phys. Rep. 292, 131 (1998).

[26] I.I. Gontchar and L.A. Litnevsky, Z. Phys. A 359, 149 (1997).

[27] A.V. Karpov, P.N. Nadtochy, D.V. Vanin, G.D. Adeev, Phys. Rev. C 63, 054610 (2001).

[28] P.N. Nadtochy, E. Vardaci, A. Di Nitto, A. Brondi, G. La Rana, R. Moro, M. Cinausero, G. Prete, N. gelli, F. Lucarelli, Phys. Lett. B 685, 258 (2010).

[29] P.N. Nadtochy, A.V. Karpov, G.D. Adeev, Yad. Fiz. 65, 832 (2002) [Phys. At. Nucl. 65, 799 (2002)].

[30] P.N. Nadtochy, A.V. Karpov, D.V. Vanin, and G.D. Adeev, Yad. Fiz. 66, 1240 (2003) [Phys. At. Nucl. 66, 1203 (2003)].

[31] I.I. Gontchar, A.E. Gettinger, L.V. Guryan, W. Wagner, Yad.Fiz. 63, 1778 (2000); Phys.Atomic Nuclei 63, 1688 (2000).

[32] I.I. Gontchar, M.V. Chushnyakova, N.E. Aktaev, A.L. Litnevsky, E.G. Pavlova, Phys. Rev. C 82, 064606 (2010).

[33] C. Schmitt, J. Bartel, A. Surowiec, K. Pomorski, Acta Phys. Pol. 33, 431 (2002)

[34] P.N. Nadtochy, A. Kelić, K.-H. Schmidt, Phys. Rev. C 75, 064614 (2007).

[35] M.R. Pahlavani and D. Naderi, Phys. Rev. C 83, 024602 (2011).

[36] K. Pomorski, et al., Nucl. Phys. A 679, 25 (2000).

[37] C. Schmitt, J. Bartel, A. Surowiec, K. Pomorski, Acta Phys. Pol. 34, 1651 (2003).

[38] E. Vardaci et al., Proceedings of the Fourth International Workshop on Nuclear Fission and Fission-Product Spectroscopy, AIP Conf. Proc. Volume 1175, 57 (2009) (DOI:10.1063/1.3258266).

[39] K. Mazurek, J.P. Wieleczko, C. Schmitt, G. Ademard, P.N. Nadtochy, Proceedings of the Scientific Workshop on Nuclear Fission Dynamics and the Emission of Prompt Neutrons and Gamma Rays, 27-29 Sept. 2010, Sinaia, Romania.

[40] V.V. Sargsyan, Yu.V. Palchikov, Z. Kanokov, G.G. Adamian, and N.V. Antonenko, Phys. Rev. A 75, 062115 (2007).

[41] H. Goutte, J.F. Berger, P. Casoli, and D. Gogny, Phys. Rev. C 71, 024316 (2005).

[42] Y. Abe, S. Ayik, P.-G. Reinhard, E. Suraud, Phys. Rep. 275, 49 (1996)

[43] H. Kramers, Physica 7, 284 (1940).

[44] G.D. Adeev, A.V. Karpov, P.N. Nadtochy, D.V. Vanin, Phys. Part. Nucl. 36, 712 (2005).

[45] P.N. Nadtochy, G.D. Adeev, A.V. Karpov, Phys. Rev. C 65, 064615 (2002).

[46] P.N. Nadtochy, A. Brondi, A. Di Nitto, G. La Rana, R. Moro, E. Vardaci, A. Ordine, A. Boiano, M. Cinausero, G. Prete, V. Rizzi, N. Gelli, F. Lucarelli, EPJ Web of Conferences 2, 08003 (2010), DOI:10.1051/epjconf/20100208003.

[47] P.N. Nadtochy, G.D. Adeev, Phys. Rev. C 72, 054608 (2005).

[48] E.G. Ryabov, A.V. Karpov, P.N. Nadtochy and G.D. Adeev, Phys. Rev. C 78, 044614 (2008).

[49] M. Brack, J. Damgaard, A.S. Jensen, H.C. Pauli, V. Strutinsky, C.Y. Wong, Rev. Mod. Phys. 44, 320 (1972).
[50] A.V. Ignatyuk, M.G. Itkis, V.N. Okolovich, G.N. Smirekin, A.S. Tishin, Yad. Fiz. 21, 1185 (1975) [Sov. J. Nucl. Phys. 21, 612 (1975)].

[51] K.T.R. Davies, A.J. Sierk, J.R. Nix, Phys. Rev. C 13, 2385 (1976).

[52] J. Blocki, Y. Boneh, J.R. Nix, J. Randrup, M. Robel, A.J. Sierk, W.J. Świątecki, Ann. Phys. (N.Y.) 113 (1978) 330.

[53] J.R. Nix, Nucl. Phys. A 502, 609 (1989).

[54] The original Lilita program developed by J. Gomez del Campo and R.G. Stockstad, Oak Ridge National Laboratory Report No. TM7295, 1981 (unpublished).

[55] H. Hauser and H. Feshbach, Phys. Rev. 87, 366 (1952).

[56] M.V. Borunov, P.N. Nadtochy, G.D. Adeev, Nucl. Phys. A 799, 56 (2008).

[57] W.D. Myers, W. J. Świạtecki, Nucl. Phys. A 601, 141 (1996).

[58] G. Ademard et al., accepted to Phys. Rev. C.

[59] G.G. Chubaryan et al., Yad. Fiz. 56, 3 (1993) [Phys. At. Nucl. 56, 286 (1993)].

[60] A.Ya. Rusanov, M.G. Itkis, V.N. Okolovich, Phys. At. Nucl. 60, 683 (1997).

[61] U.L. Businaro and S. Gallone, Nouvo Cim. 1, 629 (1955).

[62] P.M. Evans et al., Nucl. Phys. A 526, 365 (1991).

[63] A.J. Sierk, Phys. Rev. Lett. 55, 582 (1985).

[64] L.G. Sobotka, M.A. McMahan, R.J. McDonald, C. Signarbieux, G.J. Wozniak, M.L. Padgett, J.H. Gu, Z.H. Liu, Z.Q. Yao and L.G. Moretto, Phys. Rev. Lett. 53, 2004 (1984).

[65] R.K. Gupta, M.K. Sharma, S. Singh, R. Nouicer, C. Beck, Phys. Rev. C 56, 3242 (1997) and references therein

[66] P.D. Shilding et al., Phys. Rev. C 74, 064603 (2006).

[67] K. Pomorski, B. Nerlo-Pomorska and J. Bartel, Int. Jour. Mod. Phys. E 16, 566 (2007).

[68] J.P. Lestone and S.G. McCalla, Phys. Rev. C 79, 044611 (2009).

[69] C. Schmitt, K.-H. Schmidt, A. Kelic, A. Heinz, B. Jurado, and P.N. Nadtochy, Phys. Rev. C 81, 064602 (2010).

[70] D. Mancusi, R.J. Charity, and J. Cugnon, Phys. Rev. C 82, 044610 (2010).

[71] D. Ward, R.J. Charity, D.J. Hinde, J.R. Leigh, J.O. Newton, Nucl. Phys. A 403, 189 (1983).

[72] J.O. Newton, D.J. Hinde, R.J. Charity, J.R. Leigh, J.J.M. Bokhorst, A. Chatterjee, G.S. Foote, S. Ogaza, Nucl. Phys. A 483, 126 (1988).

[73] E.G. Ryabov, A.V. Karpov and G.D. Adeev, Nucl. Phys. A 765, 39 (2005).

[74] V.E. Viola, K. Kwiatkowski and M. Walker, Phys. Rev. C 31, 1550 (1985).

[75] T.K. Ghosh et al., Phys. Rev. C 79, 054607 (2009).

[76] E. de Goes Brennand, PhD thesis (2001), Université Libre de Bruxelles, Belgium, and Nukleonika 43, 241 (1998).

[77] K. Pomorski, J. Bartel, J. Richert, K. Dietrich, Nucl. Phys. A 605, 87 (1996).

[78] F.A. Ivanyuk and K. Pomorski, Phys. Rev. C 79, 054327 (2009). 